\title{
Wavelet Analysis and Lognormal Distributions in GRBs
}

\author{
Kevin J. Hurley, Brian McBreen, Fergus Quilligan, Matt Delaney \\ and Lorraine Hanlon \\ Physics Dept., University College, Dublin 4, Ireland.
}

\begin{abstract}
A wavelet analysis has been performed on 80 intense gamma-ray bursts (GRBs) from the BATSE 3B catalog with durations longer than 2 seconds. The wavelet analysis applied novel features developed for edge detection in image processing and this filtering process was used to extract a fit to the irregular GRB profile from the background. A straightforward algorithm was subsequently used to identify statistically significant peaks in this profile. The areas and FWHM of 270 peaks that were characterised as isolated were found to be consistent with lognormal distributions. The distribution of time intervals between peak maxima for all 963 identified peaks in the GRBs is also presented.
\end{abstract}

\section{INTRODUCTION}

The recent significant discoveries of fading $\mathrm{x}$-ray emission [1] have resulted in the first identification of radio and optical counterparts to GRBs $[2,3]$. The optical spectrum of one counterpart (GRB 970508) has yielded a value of $\mathrm{z}=0.835$ [4].

GRBs produce an immense amount of energy $\left(\sim 10^{51} \mathrm{ergs}\right)$ which must be emitted by a medium with highly relativistic velocities $(\gamma>100)$. Most GRBs are highly variable with a variability scale much smaller than their overall durations and there is considerable debate as to the cause of the variability, which may be due to internal or external shocks [5-7].

GRBs generally have complex time profiles and previous work [8] has indicated that some parameters are consistent with lognormal distributions e.g. durations of GRBs and time intervals between peaks. BATSE bursts with multiple peaks were also analysed and the applicability of lognormal distributions in GRBs was confirmed [9]. Other techniques have also been used to profile the time structure of GRBs [10-12]. The results presented here on a large sample of BATSE GRBs were obtained using wavelets. 
Trigger Number 1606
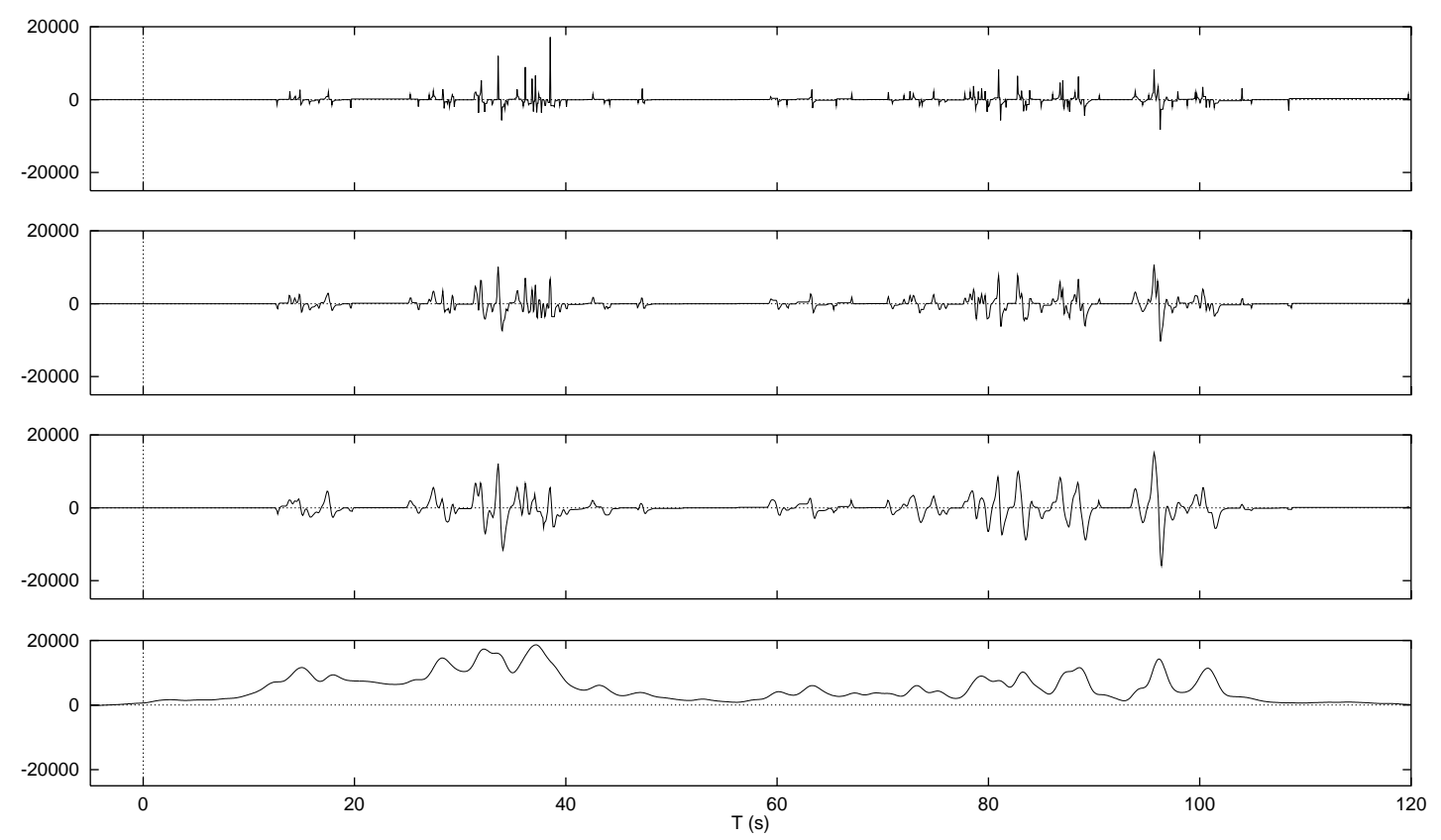

FIGURE 1. A wavelet transformation representation of GRB 920513 (BATSE trigger 1606) on three scales and the remaining low frequency signal. Note that the extreme values in the transform correspond to the edges in the signal and the signal can be reconstructed from just these extreme values [13]. This fact allows the removal of noise structure by removing the maxima and minima corresponding to the noise edges before the signal reconstruction [14].

\section{DATA PREPARATION}

The data was obtained from the BATSE 3B catalogue. A subset of this catalogue was selected based on criteria used by Norris and co-workers [11], which gave a sample of 87 long bright bursts $\left(T_{90}>2 \mathrm{~s}\right.$ and $\left.P_{256 \mathrm{~ms}}>4.6 \mathrm{ph} \mathrm{cm}^{-2} \mathrm{~s}^{-1}\right)$. The start and end times for each burst were identified by eye and a margin of 10 seconds added. Two sections of duration $30 \mathrm{~s}$, one at $-50 \mathrm{~s}$ from the start and the other at $+20 \mathrm{~s}$ from the end were used to provide a linear interpolation for background subtraction.

From the original sample of 87 bursts, a smaller sample of 80 background subtracted GRBs suitable for denoising (those with no data-gaps) were processed with a multiscale edge detection wavelet denoising routine [13-16].

Following on the wavelet denoising of the original GRB, a method was devised to identify the peak structures within the burst. Starting at the beginning of the burst, each peak was examined for minima on both sides which were separated from the maximum by more than a chosen significance level. If the search for minima failed on this peak it was rejected and the search continued. The algorithm was 


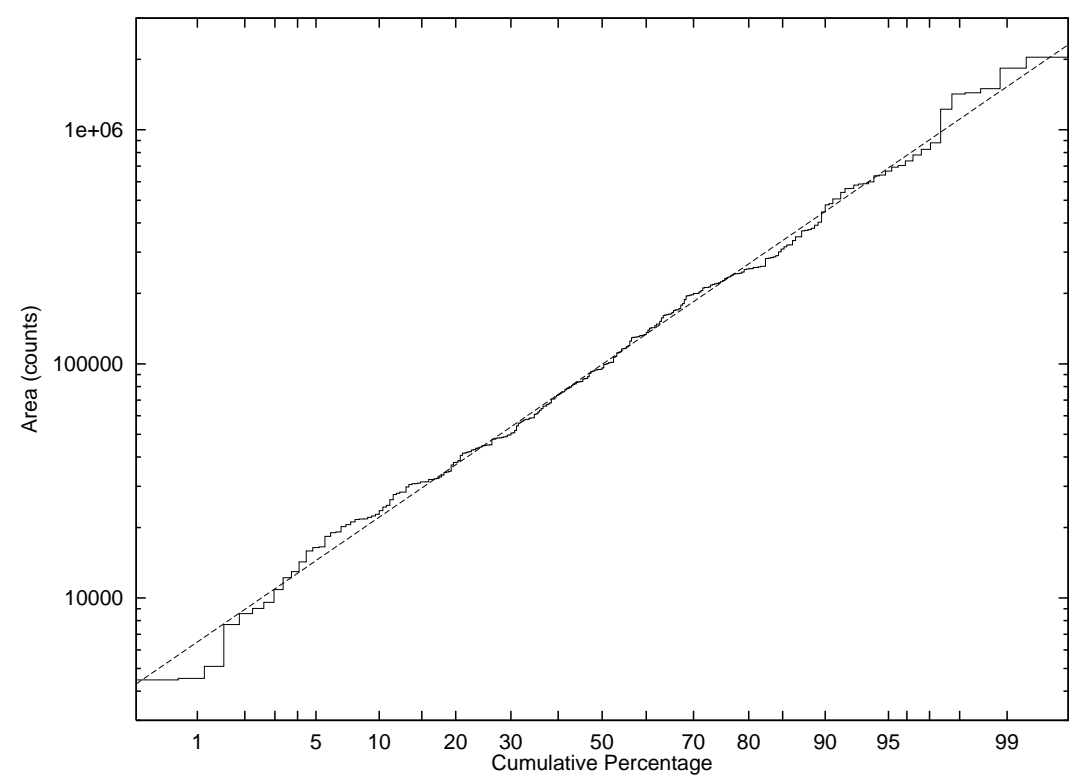

FIGURE 2. The distribution of the areas of 270 peaks that were isolated at the $66 \%$ level (see text). The dashed line is a very acceptable lognormal fit to the data (see Table 1).

designed so that the lowest minima were selected for each maxima. The analysis was extended to allow the identification of peaks which are well separated or isolated. The fraction of the total height which was above the higher minimum for every peak was calculated and then a threshold was applied on this fraction above which peaks were considered isolated.

\section{RESULTS}

Having identified peaks in the GRB time profiles, certain key characteristics of the data were examined. In particular the time interval between peaks $(\Delta T)$, the area under peaks (measured as total counts above background while the signal is above the height threshold for isolation) and the full width at half maximum (FWHM) were investigated. The distributions of each of these properties (Figures 2 and 3) was tested for compatibility with the lognormal distribution. The results of the statistical tests are presented in Table 1. It should be noted that the peak area and FWHM are quite compatible with the lognormal distribution.

\section{CONCLUSIONS}

A comprehensive profile of the temporal properties of intense GRBs has now been provided by the wavelet method. Many properties of GRBs are consistent with lognormal distributions: 


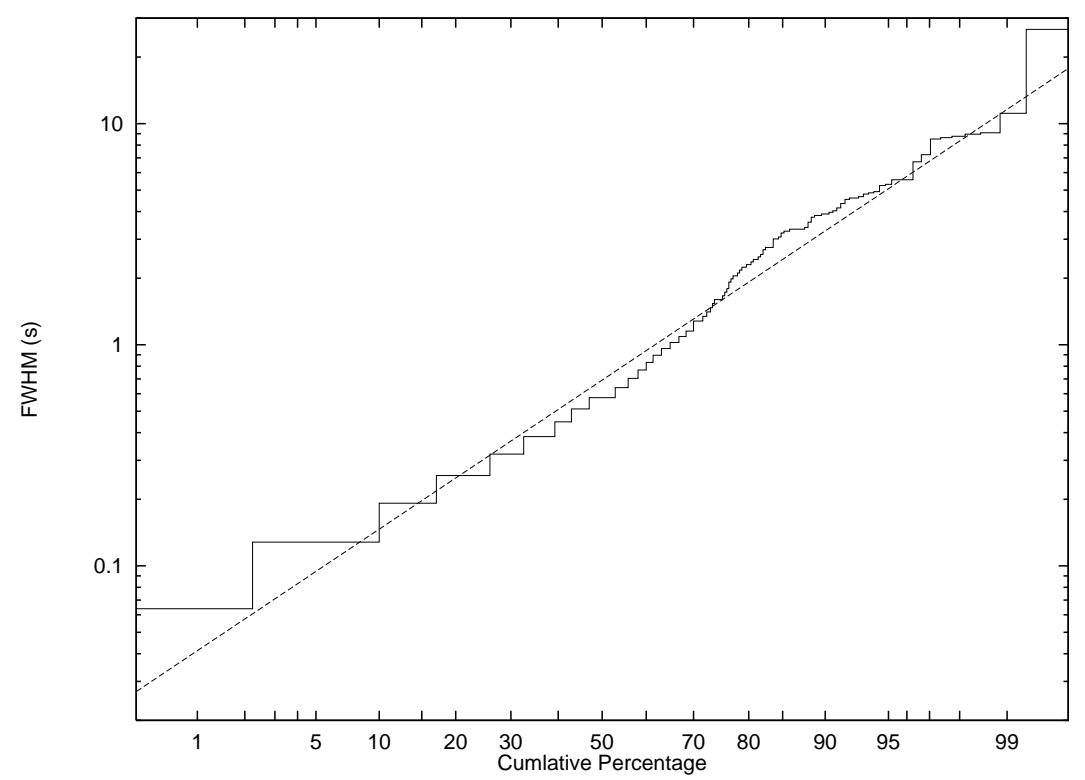

FIGURE 3. The FWHM distribution for the same peaks as in Figure 2. Again the dashed line is a lognormal fit to the data.

- The bimodal duration distribution of GRBs is consistent with two lognormal distributions [8].

- The area of the peaks is consistent with a lognormal distribution.

- The FWHM of the peaks is consistent with a lognormal distribution.

- The time intervals between peaks in GRBs as measured with this technique does not seem to be consistent with a lognormal distribution but important selection effects have not been considered.

These results are in good agreement with previous work $[8,9]$. These results provide an alternative description of GRB profiles to that of the peak-aligned stretched exponentials [10-12]. The data presented here also provide considerable constraints on models that attribute the variability to interactions with an external medium or to an internal origin that reflects the activity of the central engine.

TABLE 1. Summary of goodness of fit tests for lognormal distributions. $\chi_{2 \sigma}^{2}$ is the $2 \sigma$ confidence limit of the reduced $\chi^{2}$ value $\left(\chi^{2} / \nu\right)$ with $\nu$ degrees of freedom.

\begin{tabular}{lcllrl}
\hline \multicolumn{1}{c}{ Dist. } & Figure & $\mu$ & $\sigma$ & $\chi_{\nu}^{2} / \nu$ & $\chi_{2 \sigma}^{2}$ \\
\hline Area & 2 & 11.51 & 1.17 & 1.033 & 1.326 \\
FWHM & 3 & -0.368 & 1.212 & 1.276 & 1.306 \\
$\Delta \mathrm{T}$ & 4 & 0.05 & 1.0 & 4.119 & 1.326 \\
\hline
\end{tabular}




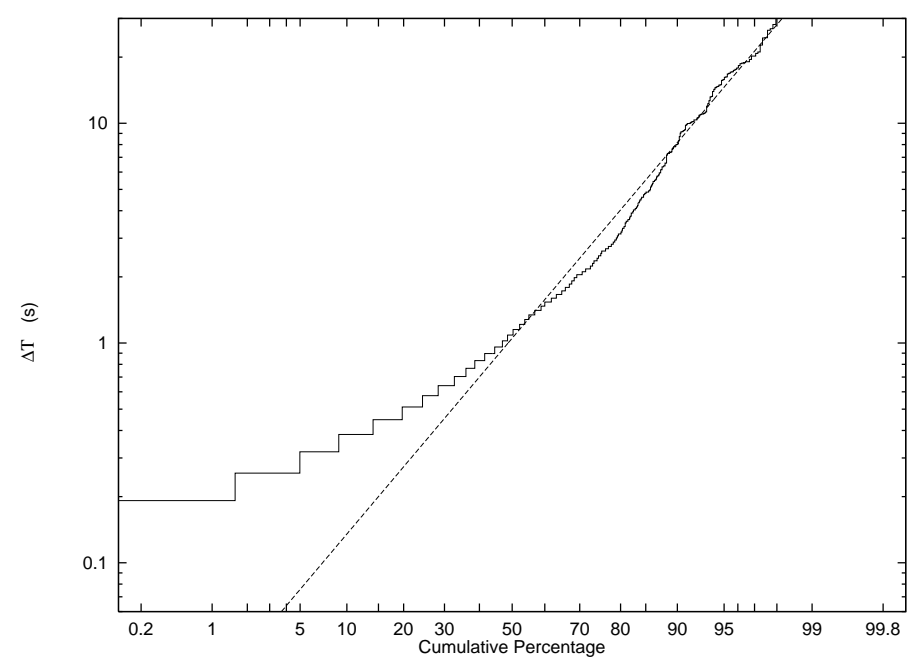

FIGURE 4. The distribution of time intervals between the 963 peaks identified in $80 \mathrm{GRBs}$. The dashed line indicates a candidate lognormal fit. The departure of the fit at short time intervals is strongly influenced by the limited resolution of the data [17].

\section{REFERENCES}

1. Costa, E. et al., 1997, Nat., 387, 783

2. Frail, D., et al., 1997, IAU Circular 6662

3. van Paradijs, J., et al., 1997, Nat., 386, 686

4. Metzger, M.R., et al., 1997, Nat., 387, 878

5. Chiang, J. \& Dermer, C.D., 1997, astro-ph/9708035

6. Panaitescu, A. \& Mészaros, P., 1997, astro-ph/9703187

7. Sari, R. \& Piran, T., 1997, astro-ph/9701002

8. McBreen, B., Hurley, K.J., Long, R. \& Metcalfe, L., 1994, MNRAS, 271, 662

9. Li, H. \& Fenimore, E.E., 1996, ApJ, 469, L115

10. Mitrofanov, I., 1995, Ap \& Space Sci., 231, 103

11. Norris, J.P. \& Svensson, R., 1996, ApJ, 459, 393

12. Stern, B. E. \& Svensson, R., 1996, ApJ, 469, L109

13. Mallat, S.G. \& Zhong, S., 1992, IEEE Trans. Patt. Anal. Mach. Intell. 7, 710

14. Mallat, S.G. \& Hwang, W.L., 1992, IEEE Info. Theory, 38, 617

15. Young, C.A., Meredith, D.C. \& Ryan, J.M., 1995, Ap. \& Space Sci. 231, 119

16. Daubechies, I., 1992, Ten Lectures on Wavelets, SIAM, Philadelphia

17. Hurley, K.J. et al., 1997, in preparation 